Wesley Y. Leonard*

\title{
Musings on Native American language reclamation and sociolinguistics
}

https://doi.org/10.1515/ijsl-2020-2086

\begin{abstract}
Sociolinguistic approaches to Native American languages are best conducted as part of a project of "language reclamation," argues Wesley Y. Leonard. He discusses how framings of Indigenous languages as "endangered," while in some ways well-intentioned, replicate the distance of language communities from scholarly research. An emphasis on reclamation - "efforts by Indigenous communities to claim the right to speak their heritage languages" highlights the role of the community members in the production of knowledge on and the revival of Native American languages.
\end{abstract}

Keywords: language reclamation, sociolinguistics, language ideology, decolonization, expanding Linguistics

Following protocols of my tribal nation, the Miami Tribe of Oklahoma, I begin by introducing myself: Wesley Leonard weenswiaani. niila myaamia. (My name is Wesley Leonard. I am Miami.) I was greatly influenced by my Miami grandfather, who emphasized that research could support the reclamation of our language, called myaamiaataweenki or just myaamia, which was a sleeping language for about 30 years and wrongly deemed "extinct" by linguists who did not acknowledge my community's process of learning myaamia from archival records. In response, I became a linguist with a focus on decolonial approaches to addressing language shift, which usually refers to a community's replacement of its heritage language with the language of a social dominant group, but which I aim to show can also entail a community's return to its heritage language. As part of this goal, I have been developing a framework of language reclamation to describe and theorize efforts by Indigenous communities to claim their right to speak their heritage languages and to set associated goals in response to community needs and perspectives.

*Corresponding author: Wesley Y. Leonard, University of California, Riverside, USA, E-mail: wesley.leonard@ucr.edu 
I recall an interview I had many years ago for a faculty position in Linguistics, ${ }^{1}$ for which I had provided a similar introduction in my application materials. The search committee expressed concern that they did not know where to place me, and mentioned some subfields of Linguistics that language reclamation apparently didn't fall into. For example, I wasn't a phonetician or phonologist, despite my engagement with sounds and sound patterns. I wasn't a morphologist, even though analysis of word formation is important in language reclamation situations. And so on.

After some more discussion about my research focus, the search committee decided I must be a sociolinguist. I could sense the relief in the room when I could finally be categorized, my work understood and evaluated with respect to a named academic tradition.

I share this anecdote because it raises general questions about how the existence and scope of academic fields can drive the ways knowledge is produced and received, as well as specific questions about what Sociolinguistics is, and by extension what it has been and can become. Looking back on the interview, I speculate that sociolinguists' focus on addressing injustices through language research - as demonstrated by the large body of scholarship that shows the value and legitimacy of stigmatized language varieties - led to my reclamation work being understood similarly. Still, language reclamation is a broad enterprise that exceeds even Linguistics as a whole, let alone any of its named subfields. The recent history of linguists' engagement with Native American and other Indigenous language issues reveals significant room for disciplinary expansion and decolonization across Linguistics, including in Sociolinguistics.

\section{1 “Endangered languages" as an important, but colonially-framed, topic in Linguistics}

A major change has occurred in Linguistics since the 1990s, when I first became involved in the field as a student: Indigenous language shift used to be a peripheral topic, but it has become progressively prominent. Many linguists identify the 1992 "Endangered Languages" series of articles in Language (Hale et al. 1992), the flagship journal of Linguistics, as pivotal to the legitimization of language endangerment as an area of disciplinary inquiry (though several

1 I capitalize disciplinary names to capture that they are specific institutions created by specific people. 
grassroots and some academic efforts had started earlier). Also in 1992, the Linguistic Society of America established the Committee on Endangered Languages and Their Preservation, and has since made several official statements about the importance of Indigenous languages. This year, there are several special events associated with the United Nations' declaration of 2019 as the International Year of Indigenous Languages. Language shift in Indigenous communities is increasingly addressed in academic publications, with journals like Language Documentation \& Conservation (established in 2006 and first published in 2007) recognized as outlets for such work. Language endangerment issues have also become part of Linguistics 101, the topic now a standard chapter in general linguistics textbooks.

Though the growing embrace of language endangerment research since the early 1990s is positive, it nonetheless remains clouded by colonial logics and structures. One major example is that Linguistics greatly emphasizes endangered language documentation over reclamation, often echoing earlier discourses of salvage ethnography by framing the need for documentation as salvaging Indigenous languages ahead of their assumed "death." A rationale frequently provided for documentation is that linguistic theory requires data from diverse languages; endangered languages must thus be documented to ensure this data will remain available for linguists. Creation of this documentation for the heritage community is touted but arguably secondary, as evidenced by documentation projects whose materials are organized in linguists' categories, framed around theoretical issues, and/or inaccessible to community practitioners. Through my lived experiences as a Miami person, this comes across as exploitation of Indigenous peoples' resources to benefit the dominant group.

While language conferences and workshops for Indigenous language communities are extremely valuable, the prestige within the academy of a given conference varies inversely with its focus on Indigenous community practitioners. Prestigious professional Linguistics conferences welcome papers about Indigenous languages, but it is common for these to focus exclusively on grammatical phenomena, with little to no mention of social contexts, language communities, or researchers' positionalities. Outcomes of the 2019 International Year of Indigenous Languages remain to be seen, but the focus in Linguistics on leveraging the International Year toward research on Indigenous languages, rather than addressing the structures that contribute to language "disappearance" (a term also used by the United Nations), is revealing. Though "language endangerment" is now a chapter in introductory textbooks, it tends to appear in the second half of these books and as a discrete topic, not as the overarching issue that it is for many language communities. 
Sociolinguistic scholarship has been instrumental for identifying and developing tools to address these issues. For example, in response to linguists and language community members alike searching for "authentic" speakers of Indigenous languages, sociolinguistic work elucidates how speakerhood gets idealized and imagined through ideological frames, which often emerge from colonial logics (see, for example, Bucholtz 2003; Adkins 2014; Meek 2014). With its recent focus on populations that experience oppression through intersecting variables such as race, gender, sexuality, religion, and political status, sociolinguistic scholarship collectively provides a general critical consciousness that facilitates intervention in Native American community language issues, which almost always involve multiple social variables and power dynamics. This critical lens guided my early scholarly work, which challenged the use of the term "extinct” for languages that are not spoken but have potential for future use, as was the case with myaamia during its 30-year dormancy.

The value of these interventions noted, several limitations remain in Sociolinguistics, which operates within and draws from a largely colonial academic industrial complex. Marginalized language communities are still often framed in othering ways that erase the potential for these community members to be viewed as scholars in a given field, for example, through questions such as "how should (socio)linguists work with Indigenous communities?," which sets up "(socio)linguists" and "Indigenous communities" as separate entities. Sociolinguistics' intellectual foundations operate similarly. Although there is a body of scholarship that reports on Native American language communities, analyses within this work are usually crafted through non-Indigenous knowledge systems and disseminated through non-Indigenous norms, to non-Indigenous audiences. There is very little "regular" linguistic scholarship (i. e. research that isn't specifically about Native American languages and people) framed through Native American protocols and ways of knowing.

\section{Sociolinguistic frontiers - learning from and contributing to language reclamation}

Such limitations are not inherent. I find the term "frontier" useful, because it draws attention not only to the limits of current knowledge within an academic field, but also to why such limits exist, and thus to the interventions that can remove them. 
For Sociolinguistics to truly engage with Native American and other Indigenous experiences, the broader discipline of Linguistics' colonial history (Errington 2008) warrants more attention, particularly with respect to how it has guided foundational disciplinary definitions and assumptions. Native Americans continue to criticize how Linguistics privileges Western epistemologies and research practices that "dissect" Native American languages in harmful ways, rendering them objects of analysis whose primary value lies in how their grammatical structures contribute to "our knowledge" (where "our" refers to linguists). The unit of analysis in contemporary Linguistics is often "language," but as a lexicogrammatical object, not as Indigenous communities tend to experience it. Sociolinguistics, despite its focus on language use and beliefs, also often privileges academic views of language by reducing community definitions to "ideologies" - itself an ideologically-driven process that occurs within power relations - without adequate reflection on how concepts such as language come to be defined in scientific contexts. Language reclamation employs this critical insight but shifts the norm to privilege local definitions of language such as "how a community connects to each other and how they express ... themselves and their culture to each other," which comes from a Miami language teacher. Other Native American views include "language is us," "language is power," and that language is integral to Indigenous laws of reciprocity. I imagine a future Sociolinguistics in which Native American and other community definitions of language will be employed as foundational analytical bases (i. e. to frame research) rather than as just topics of analysis.

A related issue emerges regarding the general assertion made about Linguistics: that the discipline is descriptive, not prescriptive - a claim that even limited engagement with language reclamation quickly demonstrates to be a distortion. This commonly articulated assertion about Linguistics misses how linguistic description has inherent prescriptive potential. This occurs because it is people who create and disseminate linguistic work, and people exist in a complex environment of power relations. In contexts of language reclamation that draw on linguistic research products, such as grammars and dictionaries, a common scenario is that these resources have been produced by credentialed linguists who are not Indigenous, and enjoy relatively high social status. Meanwhile, the prototypical users of these products are Indigenous community members whose knowledge systems are not recognized within colonial social structures as valid intellectual orientations. In such contexts, descriptions created by linguists easily come across as fixed truths rather than what they actually are - analyses by specific people who are operating in a specific scientific tradition. Crucial to consider here is Hill's (2002) and others' observation that experts' rhetoric can promote, elevate, and impose worldviews 
that align with those specialists' categories and value systems. When scholars fail to be reflexive about their own positionalities and disciplinary structures, which contain racism, sexism, colonialism, and other -isms, injustices are easily perpetuated - even by those committed to social justice.

Sociolinguistics and the related field of Linguistic Anthropology are spearheading awareness of this phenomenon, but arguably still have a long way to go. Nevertheless, I imagine a future in which not only the prescriptive potential of "description" is fully recognized, but also where the problematic power structures that anchor this potential have themselves been dismantled. I do not claim this will be an easy task, but add that research also documents how "impossible" social changes are in fact possible, as well as that it is often the agents who currently enjoy power that claim otherwise. I am optimistic because I witnessed my tribal nation - almost eliminated through removals, forced assimilation, and other colonial violence - bring back an "extinct" language despite most linguists' predictions to the contrary. Surely the comparatively well-resourced scholars that I know can bring about needed changes in Sociolinguistics and beyond.

\section{References}

Adkins, Madeleine. 2014. Will the real Breton please stand up? Language revitalization and the problem of authentic language. International Journal of the Sociology of Language 223. 55-70.

Bucholtz, Mary. 2003. Sociolinguistic nostalgia and the authentication of identity. Journal of Sociolinguistics 7(3). 398-416.

Errington, Joseph. 2008. Linguistics in a colonial world: A story of language, meaning, and power. New York: Wiley-Blackwell.

Hale, Ken, et al. 1992. Endangered languages. Language 68(1). 1-42.

Hill, Jane H. 2002. "Expert rhetorics" in advocacy for endangered languages: Who is listening, and what do they hear? Journal of Linguistic Anthropology 12(2). 119-133.

Meek, Barbra A. 2014. "She can do it in English too": Acts of intimacy and boundary-making in language revitalization. Language \& Communication 38. 73-82.

Article note: This essay was originally published in Items: Insights from the Social Sciences, a publication of the Social Science Research Council. Leonard, Wesley Y. "Musings on Native American Language Reclamation and Sociolinguistics." In "Sociolinguistic Frontiers." Series, Items: Insights from the Social Sciences. https://items.ssrc.org/sociolinguistic-frontiers/mus ings-on-native-american-language-reclamation-and-sociolinguistics/. Reprinted with permission. 